\title{
In vivo cardiac DTI on a widely available 3T clinical scanner: an optimized M2 approach
}

\author{
Christopher T Nguyen ${ }^{1 *}$, Zhaoyang Fan ${ }^{1}$, Yibin Xie ${ }^{1}$, Jianing Pang ${ }^{1}$, Xiaoming Bi ${ }^{4}$, Peter Speier ${ }^{5}$, Jon Kobashigawa ${ }^{2}$, \\ Debiao $\mathrm{Li}^{1,3}$
}

From 19th Annual SCMR Scientific Sessions

Los Angeles, CA, USA. 27-30 January 2016

\section{Background}

Currently, there are only two main methods to perform diffusion tensor CMR (DT-CMR) that either rely on the subject exhibiting stable, periodic RR cycle (stimulated echo [1]) or utilize specialized research scanners that have ultra-high gradient strengths (spin-echo [2]). Recent work has demonstrated that gradient moment nulling (GMN) of the second order is capable of yielding robust diffusion weighted images (DWI) [3]. To extend this work, we present a novel DT-CMR sequence prototype that utilizes a M2 GMN gradient scheme that is robust to imperfect B1 refocusing at high main fields $(\geq 3 \mathrm{~T})$. We compare this with no GMN compensation (M0) and first order GMN compensation (M1). Patients with advanced heart failure (HF) were also scanned to test its ability in a clinical setting.

\section{Methods}

Twenty healthy subjects and two HF patients were recruited and consented under Institutional Review Board. All subjects were scanned on a 3T Siemens (MAGNETOM Verio, Siemens Healthcare GmbH, Erlangen) with the following protocol: standard morphological localizers and 3 DTI scans (b30 +6 directions $\mathrm{b}=300 \mathrm{~s} / \mathrm{mm}^{2}$, free breathing prospective navigator gating, bSSFP readout, $2.7 \times 2.7 \times 8 \mathrm{~mm}^{3}$, flip angle $=90^{\circ}$, single-shot $\left.+\mathrm{MoCo}\right)$ utilizing M0 (TEprep = $35 \mathrm{~ms})$, M1 (TEprep = $46 \mathrm{~ms})$, and M2 (TEprep $=67 \mathrm{~ms}$ ). Acquisition was carried out during the quiescent period of diastole. Gradient amplitudes were set to $60.8 \mathrm{mT} / \mathrm{m}$ (two $43 \mathrm{mT} / \mathrm{m}$ max gradients simultaneously on). DTI reconstruction utilized custom software developed in Python using the DIPY library [6] to generate mean diffusivity (MD), fractional anisotropy (FA), and helix angle (HA) maps. Success rates defined by $>90 \%$ of the myocardium unaffected by motion was reported. Paired t-tests were utilized to statistically test for significance $(\mathrm{p}<0.05)$.

\section{Results}

For mildly low heart rates (HR) $(<75$ beats-per-min) in volunteers, M2 was shown to have significantly $(\mathrm{p}<0.05)$ higher success rates (93\%) than M1 (62\%) and M0 (28\%). For higher HR, M2 was still significantly $(\mathrm{p}<0.05)$ higher success rates $(57 \%)$ than M1 (23\%) and M0 (7\%), but much notably lower success than at lower HR. Among the scans with minimal motion artifacts, MD and FA were significantly $(\mathrm{p}<0.05)$ lower for $\mathrm{M} 2\left(1.3 \pm 0.2 \mu \mathrm{m}^{2} / \mathrm{ms}\right.$, $0.3 \pm 0.2)$ than M0 $\left(4.8 \pm 1.3 \mu \mathrm{m}^{2} / \mathrm{ms}, 0.8 \pm 0.6\right)$ and M1 $\left(1.8 \pm 0.2 \mu \mathrm{m}^{2} / \mathrm{ms}, 0.3 \pm 0.2\right)$ with $\mathrm{M} 2$ values being consistent with previous literature [1,2]. In HF patients $(\mathrm{HR}=80$ and 83 ), M2 alone was only capable of yielding motionartifact free MD, FA, and HA maps.

\section{Conclusions}

The proposed M2 was shown to be more motion robust than M1 and M0 compensation despite the shorter motion sensitivity periods. The proposed DT-CMR was the only method able to provide motion-free DT-CMR images in HF patients.

\footnotetext{
Authors' details

'Biomedical Imaging Research Institute, Cedars-Sinai Medical Center, Los Angeles, CA, USA. ${ }^{2}$ Heart Institute, Cedars-Sinai Medical Center, Los Angeles, CA, USA. 'Bioengineering, University of California Los Angeles, Los Angeles, CA, USA. ${ }^{4}$ MR R\&D, Siemens Healthcare, Los Angeles, CA, USA. ${ }^{5}$ Siemens Healthcare GmbH, Erlangen, Germany.
} 


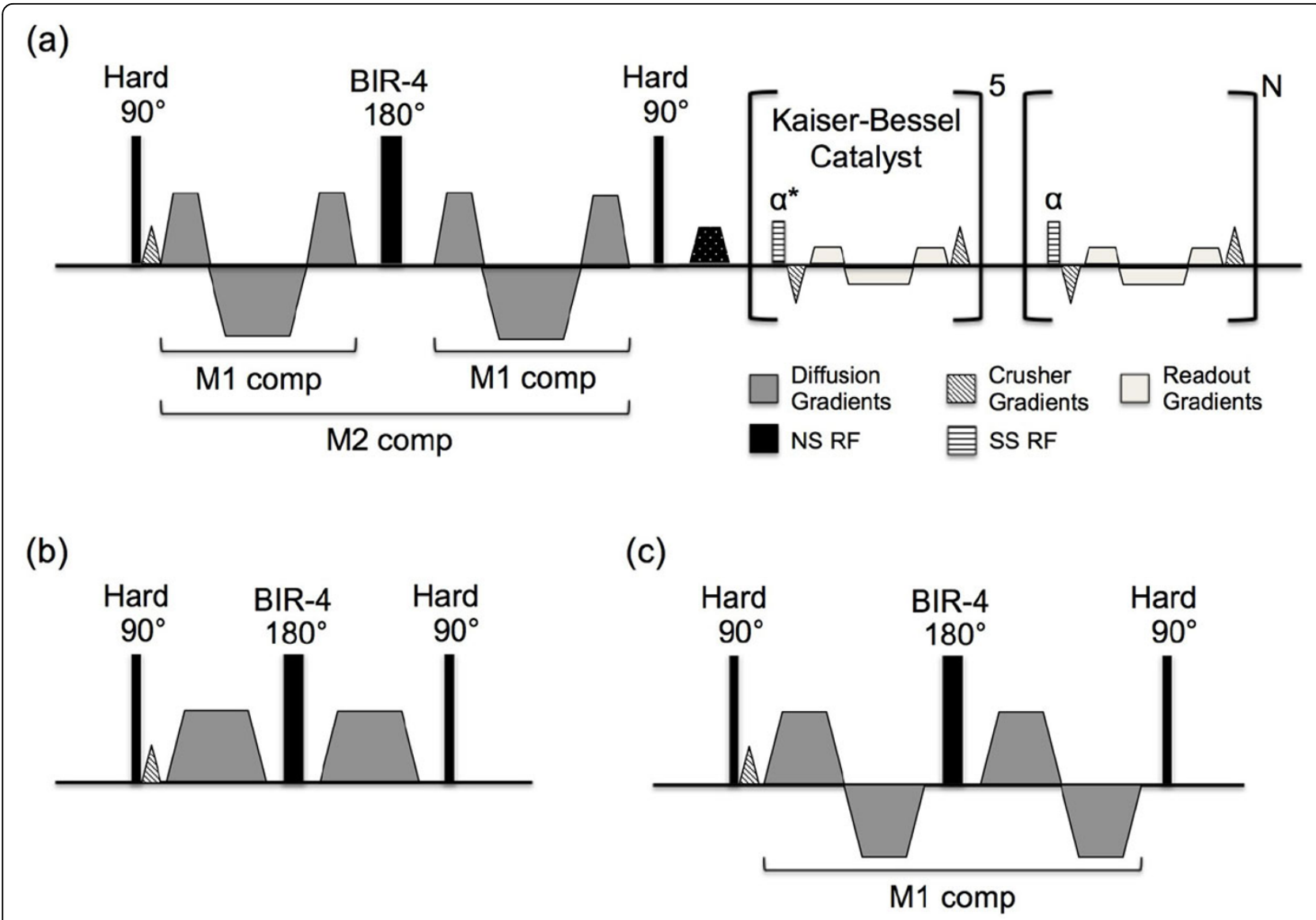

Figure 1 Pulse sequence diagram of the (a) novel dual tri-polar M2 diffusion preparation with crusher gradients [7] to provide additional robustness to $\mathbf{3}$ T B1 inhomogeneity. (b) MO and (c) M1 diffusion preparations used for comparison.

Published: 27 January 2016

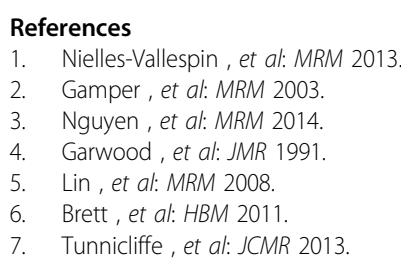

doi:10.1186/1532-429X-18-S1-018

Cite this article as: Nguyen et al:: In vivo cardiac DTI on a widely

available 3T clinical scanner: an optimized M2 approach. Journal of

Cardiovascular Magnetic Resonance 2016 18(Suppl 1):018. 


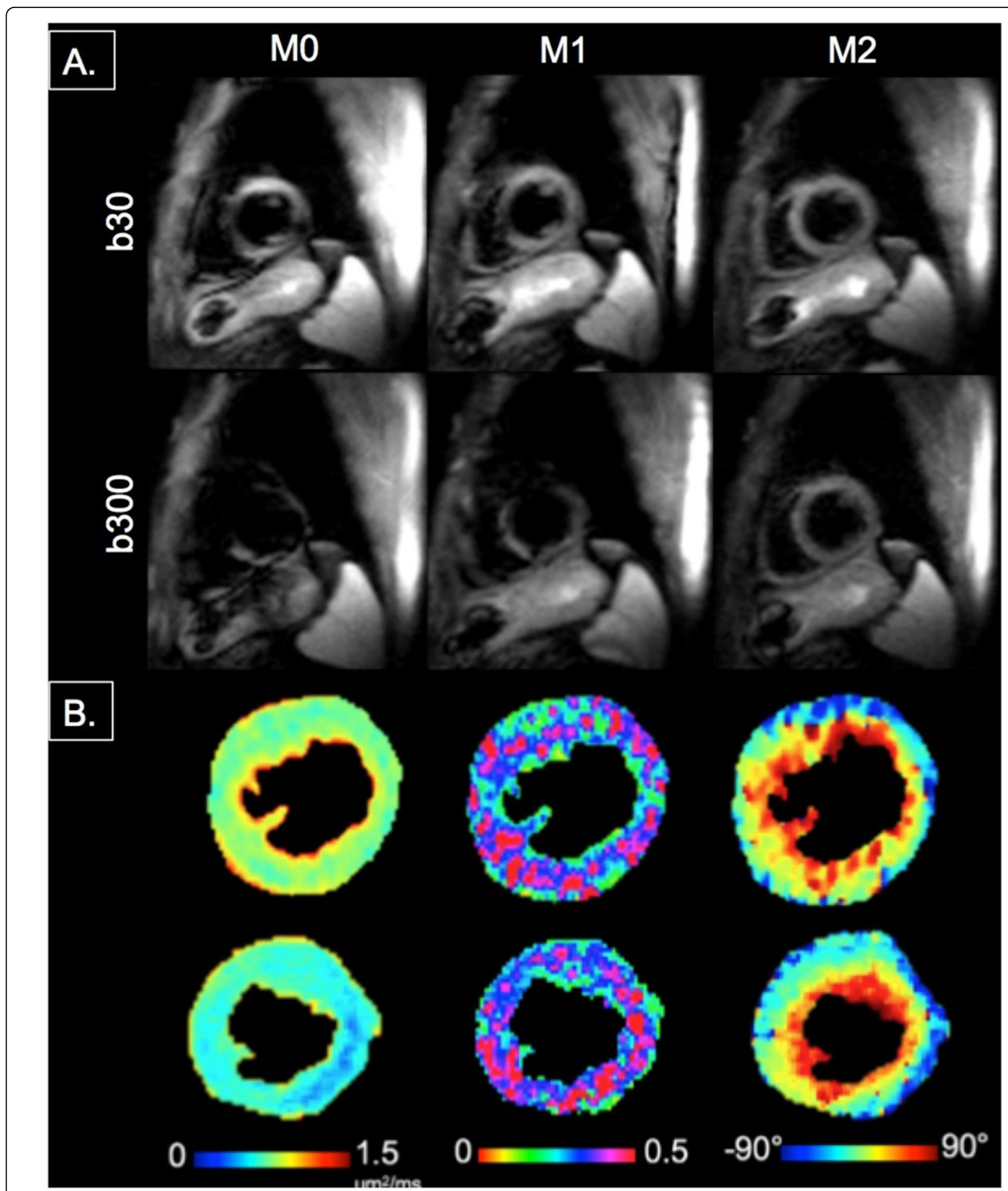

Figure 2 Representative images from a healthy volunteer $(H R=65)$ of $(A)$ least diffusion weighted (b30) and higher diffusion weighted image (b300) comparing M0, M1, and M2. (B) Representative MD, FA, and HA maps for HF patient (top row) and normal volunteer (bottom row). 